Gut, 1972, 13, 415-420

\title{
Lithogenic bile in patients with ileal dysfunction'
}

\author{
R. HERMON DOWLING, G. DUNCAN BELL, AND JUNE WHITE
}

From the Medical Research Council Intestinal Malabsorption Group, Royal Postgraduate Medical School and Hammersmith Hospital, London

SUMMARY Ileal disease or resection causes bile salt malabsorption and a reduction in the bile salt content of bile. Since cholesterol solubility requires adequate bile salt concentrations, depletion of the bile salt content of bile might, therefore, jeopardize cholesterol solubility and predispose to cholesterol gallstone formation.

To study this, we examined biliary lipid composition in 10 patients with ileal dysfunction and in 25 healthy controls.

Biliary lipid composition, as analysed in cholecystokinin-stimulated, bile-rich duodenal fluid, was shown to be representative of gallbladder bile and reproducible on repeat duodenal intubation.

Nine of the 10 patients with ileal dysfunction had an abnormal, supersaturated bile in which the limits of cholesterol solubility were exceeded, and while nine of 25 control subjects also had an unstable bile, the mean bile composition in the ileal dysfunction group was significantly different from the control population.

These studies provide a physicochemical explanation for the clinical observation that patients with ileal dysfunction have an increased incidence of gallstones.

In westernized communities the major component of most gallstones is cholesterol, and although it is virtually insoluble in water, considerable amounts of cholesterol are dissolved in normal bile because of the detergent action of bile salts and phospholipids. In turn, bile salts control both the synthesis (Nilsson and Schersten, 1970) and secretion (Swell, Bell, and Entenman, 1968; Dowling, Mack, and Small, 1971) of phospholipids in bile. Reduction in the biliary bile salt content might therefore be expected to jeopardize cholesterol solubility in bile.

It is now well known that ileal disease or resection causes bile salt malabsorption (Playoust, Lack, and Weiner, 1965) and with the resultant interruption of the enterohepatic circulation both the bile salt pool size and the bile salt content of bile are reduced (Dowling, Mack, and Small, 1970). The question arises, therefore, will ileal disease or resection impair cholesterol solubility in bile and will diseases such as Crohn's disease or radiation ileitis lead to an increased incidence of cholesterol gallstone formation?

In 1969, Heaton and Read showed that the prevalence of gallstones in 72 patients with ileal dys-

${ }^{1}$ Presented in part at a gallstones symposium organized by Weddel Pharmaceuticals at the Royal College of Physicians, London, on 28 January 1972.

Received for publication 21 March 1972. function was $32 \%$, approximately three times that of matched control populations. Similar findings have recently been reported from the United States (Cohen, Kaplan, Gottlieb, and Patterson, 1971) where $34 \%$ of 41 patients with regional enteritis were shown by cholecystography to have gallstones. However, these studies contained little information about the bile composition, nor proof that the stones were indeed cholesterol gallstones.

It has recently been shown that by measuring the bile salt, phospholipid, and cholesterol content of bile, and plotting the resultant data on triangular coordinates, one may predict whether or not the limits of cholesterol solubility have been exceeded (Admirand and Small, 1968). We have used this method of expressing results to study the effect of interruption of the bile salt enterohepatic circulation on cholesterol solubility in bile. After major interruption of the bile salt enterohepatic circulation, whether produced mechanically or by ileal resection, the rhesus monkey (an animal with a bile similar in composition to that of man) secretes a bile fully saturated with cholesterol (Dowling et al, 1971)-a vulnerable situation for cholesterol solubility.

The present paper extends the studies on bile composition with a 'broken' enterohepatic circulation to patients with ileal dysfunction. Nine out of 10 patients with ileal disease or resection had a poten- 
tially lithogenic bile, supersaturated with cholesterol. It is suggested that these results provide a physicochemical explanation for the clinical observation that patients with ileal disease have an increased prevalence of gallstones.

\section{Materials and Methods}

\section{THE PATIENTS}

Clinical details of the 10 patients with ileal dysfunction are given in Table I. There were five men and five women with a mean age of 44 (range 20-81).

\section{CONTROL SUBJECTS}

The control population were healthy volunteers from the medical and technical staff, their relatives, and patients without intestinal disease who had normal gallbladder function as shown by cholecystography. There were 15 men and 10 women with a mean age of 40 (range 17-75).

\section{BILE SAMPLING}

Following an overnight fast, the duodenum was intubated and the position of the tube checked fluoroscopically. After an intravenous injection of 75 Ivy dog units of pure cholecystokinin (CCK-PZ, Professor E. Jorpes, GIH Laboratory, Karolinska Hospital, Stockholm), four to six consecutive samples of dark, concentrated bile were aspirated over a 10-20-minute period. Fresh samples were analysed before filtration for total bile salt, phospholipid and cholesterol content as previously described (Dowling et al, 1971). After analysis, the most concentrated sample for each individual was taken as the closest approximation to gallbladder bile. The results of bile composition, expressed as ratios of bile salts:phospholipids:cholesterol in moles per cent, were then plotted on triangular coordinates and, depending on whether the resultant point fell inside or outside the zone of cholesterol solubility, the bile was regarded as normal or abnormal (Admirand and Small, 1968).

\section{VALIDATION OF DUODENAL DRAINAGE AS A} BILE SAMPLING TECHNIQUE

Comparison of duodenal drainage and gallbladder bile In five patients about to undergo biliary surgery, bile composition was measured by duodenal drainage before surgery and compared with that of gallbladder bile obtained by needle puncture at the time of operation.

\section{Reproducibility}

In five other subjects, the constancy of bile composition was checked by measuring bile composition with duodenal drainage on two different occasions.

\section{ABNORMAL OR SUPERSATURATED BILE}

At the time of aspiration, although concentrated, all the bile samples were clear solutions and when centrifuged at $37^{\circ} \mathrm{C}$ or at ambient room temperature, no precipitate was seen. However, when the abnormal bile samples were frozen and thawed, a precipitate was found, suggesting that storage at $-20^{\circ} \mathrm{C}$ and subsequent thawing had provoked precipitation from a supersaturated solution.

To test this hypothesis, fresh bile samples and the supernatant bile after freezing, thawing and centrifugation were analysed and the results in five patients with the most abnormal bile plotted on triangular coordinates. In addition, after centrifugation the precipitate was extracted with chloroform: methanol (2:1) and its cholesterol content estimated.

STATISTICAL METHODS

Since bile composition was derived from the molar
Vitamin $B_{12}$ Absorption

\begin{tabular}{|c|c|c|c|}
\hline Patient & & Age & Type and Extent of Ileal Dysfunction \\
\hline 1 & $\mathbf{F}$ & 20 & Ileal Crohn's disease \\
\hline 2 & $\mathbf{F}$ & 63 & $\begin{array}{l}\text { Radiation ileitis + resection of distal } 10 \mathrm{ft}(305 \mathrm{~cm}) \text { small bowel; remnant anastomosed } \\
\text { to transverse colon }\end{array}$ \\
\hline 3 & $\mathbf{F}$ & 64 & $11 \mathrm{ft}(335 \mathrm{~cm})$ distal small bowel resected for mesenteric vein thrombosis + infarction \\
\hline 4 & $\mathbf{F}$ & 30 & $\begin{array}{l}15 \mathrm{ft}(457 \mathrm{~cm}) \text { of distal small bowel resected; jejunal remnant anastomosed to } 2 \text { in. } \\
(5 \mathrm{~cm}) \text { of ileum }\end{array}$ \\
\hline 5 & $\mathbf{F}$ & 40 & Mesenteric embolus; 6 in. $(16 \mathrm{~cm})$ jejunum anastomosed to transverse colon \\
\hline 6 & $\mathbf{M}$ & 40 & Crohn's disease, ileal exclusion + ileo-transverse anastomosis \\
\hline 7 & $\mathbf{M}$ & 81 & $\begin{array}{l}\text { Mesenteric vascular occlusion; } 14 \text { in. }(36 \mathrm{~cm}) \text { jejunum anastomosed to mid-transverse } \\
\text { colon }\end{array}$ \\
\hline 8 & $\mathbf{M}$ & 45 & Radiation ileitis; ileal bypass + ileo-transverse anastomosis \\
\hline 9 & $\mathbf{M}$ & 29 & $\begin{array}{l}\text { Crohn's disease; distal half of small bowel resected; jejunum anastomosed to transverse } \\
\text { colon + descending colostomy }\end{array}$ \\
\hline 10 & $\mathbf{M}$ & 31 & $\begin{array}{l}\text { Road traffic accident with torn mesentery and abscess formation; } 20 \text { in. }(68 \mathrm{~cm}) \text { ileum } \\
\text { resected }+ \text { ileo-ascending anastomosis }\end{array}$ \\
\hline
\end{tabular}
(Schilling Test)

$0.4 \%$ (dose alone)

$0.6 \%$ (dose alone)

$0.13 \%$ (dose alone)

$0 \%$ (dose alone)

$0 \%$ (dose alone) $0.05 \%($ dose + IF)

$0 \%$ (dose alone)

$10 \%$ (dose + IF) 
ratio of the three biliary lipids expressed as percentages, an arc sine or angular transformation was necessary to achieve homogeneity of variance before the data from control and ileal dysfunction patients could be compared. The patient population and the control group were first compared as a whole using the $F$ test to derive probability and the individual lipids in bile were then considered separately (and compared with the Student's $t$ test).

\section{Results}

VALIDATION OF DUODENAL DRAINAGE AS A BILE SAMPLING TECHNIQUE

The molar ratios of bile salts, phospholipids, and cholesterol measured in bile-rich duodenal fluid before surgery and in the gallbladder at the time of operation were plotted on triangular coordinates and the results comparing these two techniques are shown in Figure 1a.

There was little difference in bile composition as measured by these two techniques, particularly in the proportion of cholesterol-the most important factor in predicting whether or not bile is unstable in the phase diagram.

In five other subjects studied on two separate occasions (Fig. 1b) there was again little variation in bile composition. These results suggest that bile composition measured by duodenal drainage is both

BILE COMPOSITION BY:

- DUODENAL DRAINAGE

- gall bladder puncture

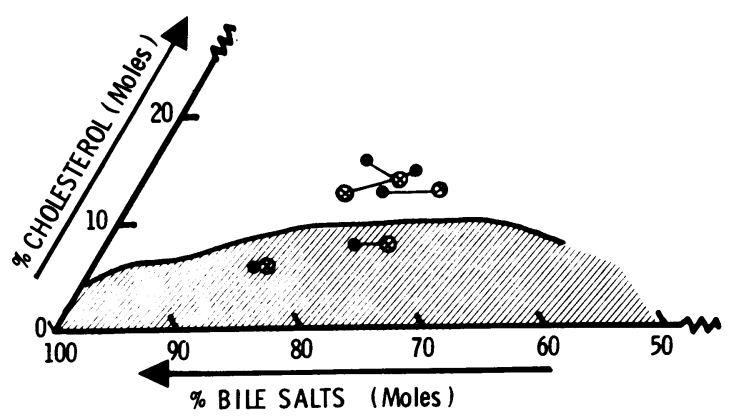

a reproducible and is a valid technique for sampling 'gallbladder bile'.

BILE COMPOSITION IN CONTROL SUBJECTS AND IN PATIENTS WITH ILEAL DYSFUNCTION The overall results of bile composition in control subjects and in patients with ileal dysfunction are given in Table II and Figure 2.

\begin{tabular}{|c|c|c|c|c|}
\hline & Bile Salts & Phospholipids & Cholesterol & \\
\hline \multirow{2}{*}{$\begin{array}{l}\text { Controls } \\
\text { Ileal } \\
\text { dysfunction }\end{array}$} & $73 \cdot 6 \pm 1 \cdot 0$ & $16 \cdot 8 \pm 0.7$ & $9 \cdot 6 \pm 0 \cdot 6$ & \multirow{2}{*}{$\begin{array}{r}F=6.796 \\
P<0.01\end{array}$} \\
\hline & $\begin{array}{l}67.4 \pm 2.0 \\
t=2.898 \\
P<0.01\end{array}$ & $\begin{array}{l}20.2 \pm 1.2 \\
t=2.525 \\
P<0.05\end{array}$ & $\begin{array}{l}12.4 \pm 0.9 \\
t=2.368 \\
P<0.05\end{array}$ & \\
\hline
\end{tabular}

Table II Biliary lipid composition in control subjects and in patients with ileal dysfunction ${ }^{1}$

${ }^{1}$ Moles \%-Mean values \pm SEM

The mean bile composition in control subjects was $73.6 \%$ bile salts ( \pm SEM $1 \cdot 0), 16 \cdot 8 \%$ phospholipids $( \pm 0.7)$, and $9.6 \%$ cholesterol $( \pm 0.6)$, and, when plotted on triangular coordinates, the mean composition fell within the micellar zone of cholesterol solubility. However, the scattergram (Fig. 2a) shows that by no means all the control subjects had a stable bile. In four of the 15 male subjects and in five of the 10 women, bile composition was repre-

Fig. 1 Validation of duodenal drainage as a technique for sampling 'gallbladder' bile. a Comparison of preoperative duodenal drainage and gallbladder bile obtained by needle aspiration at the time of operation in five patients undergoing biliary surgery.

b Reproducibility of bile composition by duodenal drainage. Results of paired studies in five subjects intubated on two separate occasions. 


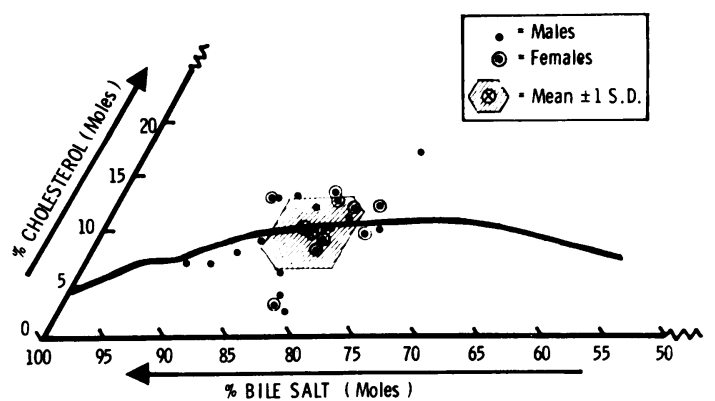

$2 \mathrm{a}$

BILE COMPOSITION IN PATIENTS WITH "BROKEN"EHC (DUODENAL DRAINAGE)

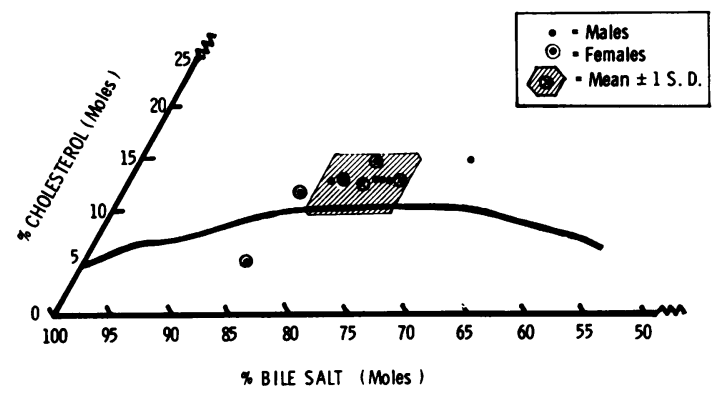

$2 \mathrm{~b}$

Fig. 2 Bile composition measured by duodenal drainage in 25 control subjects (a), and in 10 patients with a 'broken' enterohepatic circulation due to ileal dysfunction (b).

sented by points falling outside the micellar zone.

In the ileal dysfunction patients, the mean bile composition was $67 \cdot 4( \pm 2 \cdot 0) \%$ bile salts, $20 \cdot 2$ $( \pm 1 \cdot 2) \%$ phospholipids, and $12.4( \pm 1.0) \%$ cholesterol, which, when plotted on triangular coordinates, falls outside the micellar zone (Fig. 2b). In fact, nine of the 10 patients with interruption of their enterohepatic circulation had an unstable bile.

The results for the ileal dysfunction patients were statistically different from the control subjects whether the two populations were compared as a whole $(F=6.796 ; P<0.01)$ or for each individual biliary lipid-bile salts $(\mathrm{t}=2.898 ; \quad \mathrm{P}<0.01)$; phospholipids $(t=2.525 ; P<0.05)$; and cholesterol $(t=2.368 ; P<0.05)$. The difference between the two populations was most marked for males, since none of the five men with disease or resection of the ileum had stable bile, while in contrast $73 \%$ of the male control subjects had normal bile composition.

CONFIRMATION OF SUPERSATURATION IN ABNORMAL BILE

Figure 3 shows the composition of fresh bile before

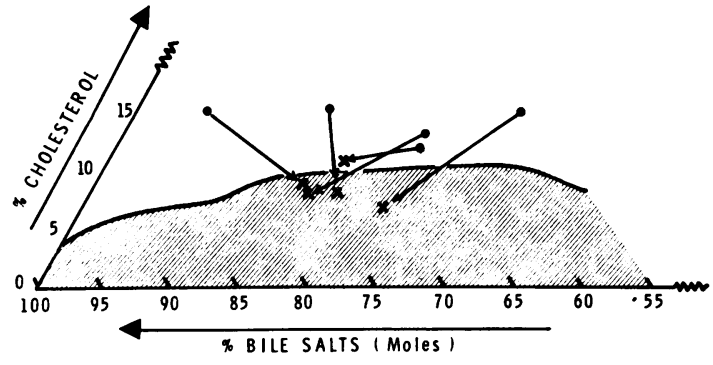

Fig. 3 Biliary lipid composition in five patients with ileal dysfunction as measured in fresh bile before filtration (solid circles) and in the supernatant of bile stored at $-20^{\circ} \mathrm{C}$ and subsequently thawed and centrifuged (crosses).

filtration, compared with supernatant bile after freezing, thawing, and centrifugation.

In all five patients with ileal dysfunction in whom this comparison was made, the composition of supernatant bile after storage was represented by points moving on or near to the boundary line of cholesterol solubility, suggesting that a supersaturated solution had become converted to a bile saturated with cholesterol. Analysis of the precipitate showed that it contained the cholesterol previously held in supersaturated solution. However, as Fig. 3 shows, not only did the proportion of cholesterol change after freezing, thawing, and centrifugation, but the ratio of bile salts : phospholipids also showed a variable degree of change, suggesting that freezing may well have precipitated substances other than cholesterol.

\section{Discussion}

These studies show that bile composition in patients with ileal dysfunction, and resultant interruption of the bile salt enterohepatic circulation, becomes unstable being supersaturated with cholesterol-a situation likely to predispose to cholesterol precipitation and gallstone formation. The present results, therefore, provide physicochemical evidence to support the clinical observation that patients with disease or resection of the ileum have an increased incidence of gallstones (Heaton and Read, 1969; Cohen et al, 1971). However, the demonstration of gallstones by plain radiographs or by cholecystography provides no information about the nature of the gallstones. (We have recently found that out of 20 patients with presumed cholesterol gallstones coming to routine cholecystectomy at Hammersmith Hospital in the past six months, three $(15 \%)$ 
had 'pigment type' stones containing only trace amounts of cholesterol ${ }^{1}$ ).

\section{REPRODUCIBILITY}

The technique used in the present study to sample 'gallbladder' bile by aspirating bile-rich duodenal fluid seems, from the results, to be a valid technique. It has previously been shown that the pattern of bile salts in bile-rich duodenal fluid is similar to that of gallbladder bile (Sjövall, 1960), and, in agreement with the present results, two recent papers (Thistle and Schoenfield, 1971; Vlahcevic, Bell, Juttijudata, and Swell, 1971) (published since this study was completed) have shown that analysis of duodenal drainage is also a reliable way of sampling the lipids of gallbladder bile.

SIGNIFICANCE OF ABNORMAL BILE

The presence of an abnormal bile, as plotted on triangular coordinates, could either mean that the bile was supersaturated with cholesterol or that it was a suspension containing micro crystals of cholesterol (Admirand and Small, 1968; Juniper and Burson, 1957). In the present studies, 'whole bile' was analysed fresh without filtration. If the gallbladder bile contains microcrystals of cholesterol, it would seem logical, at least in the first instance, to analyse the whole sample. No precipitation was found on centrifuging fresh bile and there was no difference in bile composition before and in the supernatant of fresh bile after centrifugation, suggesting that the instability was probably not due to a microcrystalline suspension. Rather, the incuction of cholesterol precipitation and the transformation of an abnormal bile into a solution saturated with cholesterol by freezing and thawing, suggests that the abnormal bile was originally a supersaturated solution. Similar observations have previously been made for rhesus monkey bile (Dowling et al, 1971) and for human bile (Thistle and Schoenfield, 1971).

The presence of a supersaturated bile does not imply that gallstones are present nor even that cholesterol precipitation will necessarily occur. It simply means that at the time of study the individual is vulnerable to cholesterol precipitation. If the primary abnormality persists, secondary factors such as stasis and nucleation or 'seeding' of the supersaturated solution, perhaps by mucopolysaccharides (Bouchier, Cooperband, and el-Kodsi, 1965) or bacteria in infected bile, may well be important in promoting precipitation and subsequent aggrega-

${ }^{1}$ The results of bile composition analysed both by duodenal drainage and by aspiration of gallbladder bile at the time of surgery have been correlated with $x$-ray and chemical analysis of gallstone composition and will be the subject of further communications (Bell, Sutor, and Dowling, unpublished observations). tion, lamellar crystalline growth, and ultimately stone formation.

The significance of an abnormal bile in control subjects is uncertain. It is clear, however, that many healthy, asymptomatic individuals who have normal cholecystograms may have abnormal bile when analysed by duodenal drainage. As a group, our patients with ileal dysfunction were significantly different from the control group, but there was, nonetheless, a $36 \%$ overlap which contrasts with the $100 \%$ separation between control and lithogenic biles reported by Admirand and Small (1968). Whether or not control subjects with abnormal biles have a basic metabolic defect causing the liver to secrete an abnormal bile, the suggested abnormality in the Pima Indians who have an extremely high incidence of cholesterol gallstones (Small and Rapo, 1970), remains to be seen. It might also be argued that those 'control' subjects with a supersaturated bile may subsequently develop gallstones, but clearly this remains conjectural.

The present results in patients with chronic interruption of the enterohepatic circulation produced by disease or resection of the ileum extend our observations in the rhesus monkey (Dowling et al, 1970). Following ileal resection, the liver responds with increased bile salt synthesis and the residual intestine shows enhanced bile salt reabsorption (Perry, White, and Dowling, 1972) but these adaptive mechanisms are both limited and inadequate to compensate for bile salt loss so that the net effect is a reduction in bile salt pool size and the relative proportion of bile salts in bile. As a result, the animals secrete a bile completely saturated with cholesterol (Dowling et al, 1971).

The mechanism for the potentially lithogenic bile in patients with ileal dysfunction is almost certainly related to interruption of the bile salt enterohepatic circulation with an attendant reduction in bile salt pool size (Dowling et al, 1970). Normally, biliary phospholipid and cholesterol secretion closely parallel the bile salt output in bile but, following chronic, and more particularly after acute interruption of the enterohepatic circulation, this intimate relationship becomes 'uncoupled'. It has been shown both in experimental animals (Dowling et al, 1971) and in man (Thureborn, 1962; Nilsson and Scherstén, 1969) that for several hours after acute interruption of the enterohepatic circulation, fistula bile consistently becomes supersaturated with cholesterol.

In common with patients who have ileal dysfunction, gallstone patients also have a diminished bile salt pool (Vlahcevic, Bell, Buhac, Farrar, and Swell, 1970; Danzinger, Hofmann, Schoenfield, and Thistle, 1971). It is suggested, therefore, that reduction in the bile salt pool size may be the key 
factor in the pathogenesis of lithogenic bile and ultimately in cholesterol gallstone formation.

Thanks are due to Miss Aviva Petrie for advice and help with statistical methods. Financial support to the MRC Intestinal Malabsorption Group (R.H.D.) and from Weddel Pharmaceuticals (G.D.B.) is gratefully acknowledged. We also wish to thank our surgical colleagues for their helpful cooperation in collecting operative samples of bile.

References

Admirand, W. H., and Small, D. M. (1968). The physicochemical basis of cholesterol gallstone formation in man. J. clin. Invest. 47, 1043-1052.

Bouchier, I. A., Cooperband, S. R., and el-Kodsi, B. M. (1965). Mucous substances and viscosity of normal and pathological human bile. Gastroenterology, 49, 343-353.

Cohen, S., Kaplan, M., Gottlieb, L., and Patterson, J. (1971). Liver disease and gallstones in regional enteritis. Gastroenterology, $60,237-245$

Danzinger, R. G., Hofmann, A. F., Schoenfield, L. J., and Thistle, J. L. (1971). Altered bile acid metabolism in patients with cholesterol cholelithiasis. (Abstr.) J. clin. Invest., 50, 24a.

Dowling, R. H., Mack, E., and Small, D. M. (1970). Effects of controlled interruption of the enterohepatic circulation of bile salts by biliary diversion and by ileal resection on bile salt secretion, synthesis and pool size in the rhesus monkey. J. clin. Invest., 49, 232-242.

Dowling, R. H., Mack, E., and Small, D. M. (1971). Biliary lipid secretion and bile composition after acute and chronic interruption of the enterohepatic circulation in the rhesus monkey. J. clin. Invest., 50, 1917-1926.
Heaton, K. W., and Read, A. E. (1969). Gallstones in patients with disorders of the terminal ileum and disturbed bile salt metabolism. Brit. med. J., 3, 494-496.

Juniper, K., Jr., and Burson, E. N., Jr. (1957). Biliary tract studies. II. The significance of biliary crystals. Gastroenterology, 32, 175211.

Nilsson, S., and Schersten, T. (1969). Importance of bile acids for phospholipid secretion into human hepatic bile. Gastroenterology, 57, 525-532.

Nilsson, S., and Schersten, T. (1970). Influence of bile acids on the synthesis of biliary phospholipids in man. Europ. J. clin. Invest., 1, 109-111.

Perry, M., White, J., and Dowling, R. H. (1972). Bile acid absorption by jejunum and colon after ileal resection in the rat. Brit. $J$. Surg., 59, 31 (Abstr.)

Playoust, M. R., Lack, L., and Weiner, I. M. (1965). Effect of intestinal resection on bile salt absorption in dogs. Amer. J. Physiol., 208, 363-369.

Small, D. M., and Rapo, S. (1970). Source of abnormal bile in patients with cholesterol gallstones. New Engl. J. Med., 283, 53-57.

Sjövall, J. (1960). Bile acids in man under normal and pathological conditions: Bile acids and steroids 73. Clin. chim. Acta, 5, 33-41.

Swell, L., Bell, C. C., Jr., and Entenman, C. (1968). Bile acids and lipid metabolism. III. Influence of bile acids on phospholipids in liver and bile of the isolated perfused dog liver. Biochim. biophys. Acta, 164, 278-284.

Thistle, J. L., and Schoenfield, L. J. (1971). Induced alterations in composition of bile of persons having cholelithiasis. Gastroenterology, 61, 488-496.

Thureborn, E. (1962). Human hepatic bile: Composition changes due to altered enterohepatic circulation. Acta chir. scand., Suppl., 303, 1 .

Vlahcevic, Z. R., Bell, C. C. Jr., Buhac, I., Farrar, J. T., and Swell, L. (1970). Diminished bile acid pool size in patients with gallstones. Gastroenterology, 59, 165-173.

Vlahcevic, Z. R., Bell, C. C. Jr., Juttijudata, P., and Swell, L. (1971). Duodenal fluid as indicator of biliary lipid composition. Amer. J. dig. Dis., 16, 797-802. 